\title{
Multiband wave filtering and waveguiding in bio-inspired hierarchical composites
}

\author{
Yanyu Chen and Lifeng Wang*
}

Department of Mechanical Engineering, State University of New York at Stony Brook, Stony Brook, New York 11794, USA

* Corresponding author. E-mail address: Lifeng.wang@stonybrook.edu (L. Wang)

\section{ABSTRACT}

We investigate the elastic wave propagation in bio-inspired hierarchical composites with nacre-like and biocalcite-like architectures. These two types of architectures consist of hard mineral and soft organic phases, which are hierarchically assembled to develop multilevel of hierarchy. We numerically demonstrate that multiple band gaps and passbands, covering an ultrawide frequency range, arise in the proposed hierarchical composites with two levels of hierarchy. We further reveal that the multilevel structural hierarchy itself is responsible for this multiband characteristic. Specifically, the low frequency band gaps in the composites with two levels of hierarchy are attributed to Bragg scattering, which are intrinsically governed by the hierarchical and periodic modulation of constituent phases at the second hierarchical level. By contrast, the multiple band gaps and passbands in high frequency ranges correspond to waveguide modes, enabling the incident wave to be either trapped inside the waveguides or efficiently transmitted through the waveguides. The findings in this paper not only shed light on the mechanisms responsible for the multiband features of bio-inspired hierarchical composites, but also offer new opportunities towards the design of compact and mechanically robust phononic crystals with the capability to effectively manipulate wave propagation.

KEYWORDS: multiband, wave filtering, waveguide, hierarchical composites, phononic crystals

\section{Introduction}

Manipulating acoustic and elastic wave propagation using rationally designed architectures has attracted increasing research interests in recent years. The periodic architectures of phononic crystals, for example, can modify phonon dispersion relations, providing avenues to tailor group velocities and hence the flow 
33 of vibrational energy [1,2]. When the structural periodicities of phononic crystals have the same order 34 of magnitude as the wavelengths of acoustic and elastic waves, multiple scatterings arise at the interfaces between constituent phases with contrast in elastic constants. This mechanism gives rise to

36 complete wave band gaps, where propagation of phonons is prohibited, irrespective of incident angles.

37 Interestingly, even if the structural periodicity of phononic crystals is interrupted by defects, it is still possible to effectively manipulate wave propagation, such as wave bending and wave splitting [3-8]. These capabilities make the perfect and defective phononic crystals particularly suitable for designing wave filters and waveguides $[4,5,7,9-12]$.

Aside from the basic requirements of wave filtering and waveguiding capabilities, a few novel attributes, including multiple band gaps, subwavelength characteristic, compact size, and outstanding mechanical performance, are highly desirable in engineering practice. Conventional phononic crystals, however, become inefficient when these properties are simultaneously pursued. Inspired by the fractal design of their counterparts in electromagnetic waves [13-16], phononic crystals with periodic fractal architectures have been proposed recently [17-19]. The rationally designed fractal architectures can give rise to multiple band gaps as well as the shifting of band gaps towards lower frequency ranges for longitudinal waves [17]. Similar to the fractal design of phononic crystals, bio-inspired hierarchical composites with multilevel structural hierarchies have been reported, which exhibit a broadband wave filtering phenomenon [20]. In addition, a multiobjective optimization method has been proposed to achieve desired wave dispersion properties, including simultaneous multiple passbands and stopbands in onedimensional layered systems $[21,22]$. These progresses indicate that the desired multiple and broad band gaps could be achieved by rationally designing the inherent architectures of phononic crystals.

This paper aims to explore the elastic wave propagation in bio-inspired hierarchical composites with nacre-like and biocalcite-like architectures. These two types of architectures consist of hard mineral and soft organic phases, which are hierarchically assembled to develop multilevel of structural hierarchy 59 (Fig.1). Guided by the finite element modeling, we show that multiple band gaps and passbands, covering an ultrawide frequency range, arise in the hierarchical composites with two levels of hierarchy.

61 In particular, low frequency band gaps, akin to the subwavelength characteristic in acoustic 62 metamaterials, exist in the hierarchical composites with two levels of hierarchy. We emphasize that the 
63 mechanisms responsible for the multiple band gaps and passbands are totally different, depending on the 64 frequency ranges of the band gaps and passbands.

\section{Numerical modeling}

\subsection{Characterization of the hierarchical composites}

The proposed bio-inspired composites have a nacre-like architecture and a biocalcite-like architecture with two levels $(N=2)$ of structural hierarchy, where $N$ is the total number of structural hierarchy level (Fig.1. (a) and (b)). In the nacre-like composite, the soft organic phase is continuous, with the hard mineral platelets dispersed in the soft organic matrix. In the biocalcite-like composite, however, the soft organic platelets are distributed in the continuous hard mineral phase. The two-dimensional periodicity at each level of the hierarchical architectures is characterized by a rhombic lattice with vectors $\mathbf{a}_{n 1}=$ $\left[\left(l_{n}+t_{n}\right) / 2, \tan \alpha_{n} \cdot\left(l_{n}+t_{n}\right) / 2\right]$, and $\mathbf{a}_{n 2}=\left[\left(l_{n}+t_{n}\right) / 2,-\tan \alpha_{n} \cdot\left(l_{n}+t_{n}\right) / 2\right]$, where $l_{n}$ is the length of the mineral platelet, $t_{n}$ is the thickness of the matrix, $\alpha_{n}$ is the lattice angle (Fig.1. (c) and (d)), and the subscript $n$ denotes the order of structural hierarchy level. In this regard, the volume fraction of the mineral phase can be defined as $v_{f n}=2 l_{n} h_{n} /\left[\left(l_{n}+t_{n}\right)^{2} \cdot \tan \alpha_{n}\right]$ for level $n=1,2$ of nacre-like composite and level $n=2$ of biocalcite-like composite; while for level $n=1$ of biocalcite-like composites, the volume fraction of

\section{2 level $n$.}

We assume the overall volume fractions of mineral phase in the composites with $N=1$ and $N=2$ levels of hierarchy are equal to $V_{f n}=0.80$. To ensure the self-similarity in each level of the composite with $N=2$ levels of hierarchy, the volume fraction of mineral phase in each level is given by $v_{f n}=\sqrt{V_{f n}}=0.894$. In addition, the lattice angle is taken as $\alpha_{n}=30^{\circ}$ in each level. We further assume that for level $n=1$ of the nacre-like and biocalcite-like composites, $l_{1}=10 \mu \mathrm{m}$ and $t_{1}=l_{1} / 50=0.2 \mu \mathrm{m}$. Considering the trade-off between accuracy and computational burden, we use four unit cells (nine layers of hard minerals) along vertical direction in each level of structural hierarchy. Then the parameters in level $n=2$ can be calculated as $l_{2}=97.69 \mu \mathrm{m}$ and $t_{2}=l_{2} / 50=1.95 \mu \mathrm{m}$ accordingly. 

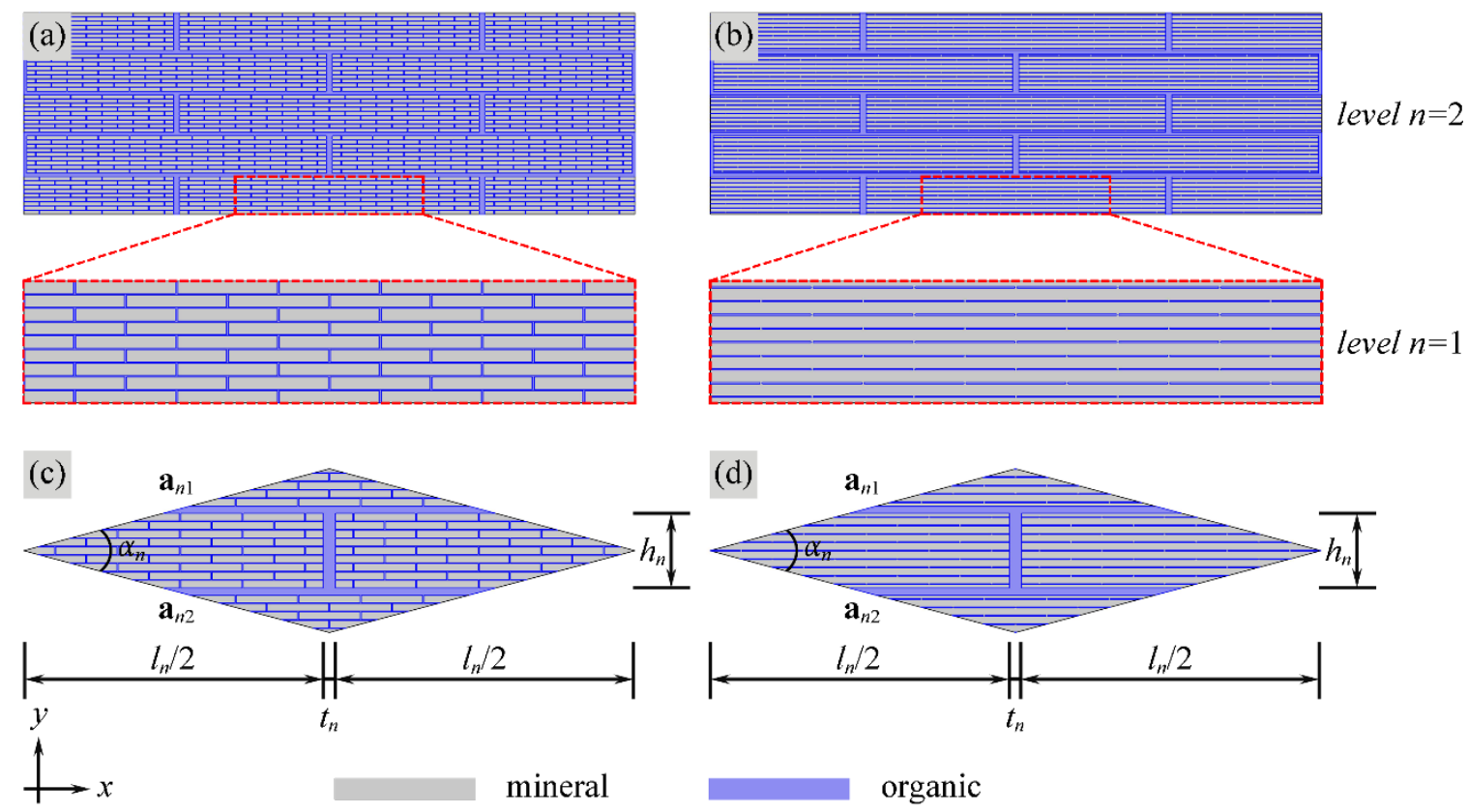

Fig.1. Schematics of the proposed hierarchical composites and the associated supercells. (a)-(b): Nacrelike and biocalcite-like hierarchical composites with two $(N=2)$ levels of structural hierarchy, where $N$ is the total number of structural hierarchy level; (c)-(d) supercells of nacre-like and biocalcite-like

97 hierarchical composites. The two-dimensional periodicity at each level is characterized by a rhombic 98 lattice with vectors $\mathbf{a}_{n 1}=\left[\left(l_{n}+t_{n}\right) / 2, \tan \alpha_{n} \cdot\left(l_{n}+t_{n}\right) / 2\right]$, and $\mathbf{a}_{n 2}=\left[\left(l_{n}+t_{n}\right) / 2,-\tan \alpha_{n} \cdot\left(l_{n}+t_{n}\right) / 2\right]$, where $l_{n}$ is the 99 length of the platelet, $t_{n}$ is the thickness of the matrix, $\alpha_{n}$ is the lattice angle, and the subscript $n$ denotes 100 the order of structural hierarchy level.

\subsection{Numerical modeling of wave propagation}

The governing equation of elastic wave propagation in the hierarchical composites can be written as

$-\rho \omega^{2} \mathbf{u}=\frac{E}{2(1+v)} \nabla^{2} \mathbf{u}+\frac{E}{2(1+v)(1-2 v)} \nabla(\nabla \cdot \mathbf{u})$

where $\mathbf{u}$ is the displacement vector, and $\omega$ is the angular frequency. $E, \nu$, and $\rho$ are the Young's modulus, the Poisson's ratio, and the density of each constituent phase, respectively. Here we assume the constituent phases of the hierarchical composites are homogeneous, isotropic and linearly elastic. Their properties are characterized by, Young's modulus $E_{m}=100 \mathrm{GPa}$, Poisson's ratio $\nu_{m}=0.30$, and density $\rho_{m}$ 
$110=2950 \mathrm{~kg} / \mathrm{m}^{3}$ for the mineral phase, and Young's modulus $E_{o}=1 \mathrm{GPa}$, Poisson's ratio $v_{o}=0.30$, and 111 density $\rho_{o}=1350 \mathrm{~kg} / \mathrm{m}^{3}$ for the organic phase [23-27].

112 The transmission spectra of the elastic wave propagation in the proposed composites are calculated by 113 performing frequency domain analyses. To model the normally incident elastic wave propagating in the 114 hierarchical composites, a harmonic vertical displacement with an amplitude of $0.01 \mu \mathrm{m}$ is applied on 115 the top surface of the composites with $1 \times 4$ supercells. Perfectly matched layers (PMLs) are applied at 116 the two ends of the homogeneous parts to prevent reflections by the scattering waves from the domain 117 boundaries [28]. In addition, periodic boundary conditions are applied on the two lateral sides of the 118 composites to model the infinite periodicity of the supercells. The transmission coefficient is defined as $119 \phi=(u+v) /\left(u_{0}+v_{0}\right)$, where $u$ and $v$ are the amplitudes of transmitted horizontal and vertical 120 displacements, respectively, and $u_{0}$ and $v_{0}$ are the amplitudes of applied horizontal and vertical 121 displacements, respectively. Since we only apply a small amplitude vertical displacement, then we have $122 u_{0}=0$, and $v_{0}=0.01 \mu \mathrm{m}$ in this study.

123 The phononic dispersion relations are constructed by performing eigenfrequency analyses. To this end, 124 Bloch's periodic boundary conditions are applied at the boundaries of the supercell such that

$$
\mathbf{u}_{i}(\mathbf{r}+\mathbf{a})=e^{i \mathbf{k} \cdot \mathbf{a}} \mathbf{u}_{i}(\mathbf{r})
$$

126 where $\mathbf{r}$ is the location vector, $\mathbf{a}$ is the lattice translation vector, and $\mathbf{k}$ is the wave vector.

127 The governing equation (1) combining with the boundary condition, equation (2), leads to the standard 128 eigenvalue problem:

$129\left(\mathbf{K}-\omega^{2} \mathbf{M}\right) \mathbf{U}=0$

130 where $\mathbf{U}$ is the assembled displacement vector, and $\mathbf{K}$ and $\mathbf{M}$ are the global stiffness and mass matrices 131 assembled using standard finite element analysis procedure, respectively. Eq. (3) is numerically solved 132 by imposing two components of wave vectors and hence yields the corresponding eigenfrequencies. The 133 phonon dispersion relations are obtained by scanning the wave vectors in the first Brillouin zone.

\section{Results and discussion}

We start by examining the transmission property of elastic wave propagation in the proposed 139 hierarchical composites. Figure 2 shows the transmission spectra of the normally incident elastic wave propagating in the nacre-like and biocalcite-like hierarchical composites with $N=1$ and $N=2$ levels of 
140 structural hierarchy. It is observed that in both composites with $N=1$ level of hierarchy, about 7 wide 141 continuous band gaps arise in the frequency range $0.4 \sim 1.5 \mathrm{GHz}$. These band gaps are attributed to Bragg 142 scattering and/or local resonances, as our previous study revealed [29]. However, when the total number 143 of structural hierarchy is increased into $N=2$, the original wide continuous band gaps degenerate into 144 multiple sharp band gaps and passbands. The most remarkable phenomenon in the transmission spectra 145 of composites with $N=2$ levels of structural hierarchy is the emergence of 4 5 new band gaps located in 146 low frequency range $0 \sim 0.3 \mathrm{GHz}$, akin to the subwavelength characteristic of acoustic metamaterials [30]. 147 These phenomena are similar to those observed in one-dimensional layered composites with multilevel 148 hierarchies and two-dimensional solid-fluid system with fractal architectures[17, 20]. The difference is 149 that these new features arising in the proposed composites are obtained by simply assembling the 150 mineral and organic phases in a hierarchical manner. Therefore, we believe that the structural hierarchy 151 itself accounts for the low frequency and multiband features of the composites with $N=2$ levels of 152 hierarchy. Specifically, the periodic arrangement of mineral and organic phases in hierarchical level $n=2$ 153 leads to the emergence of low frequency band gaps. At level $n=1$, however, the structural periodicity is 154 interrupted, resulting in multiple shape band gaps and passbands.
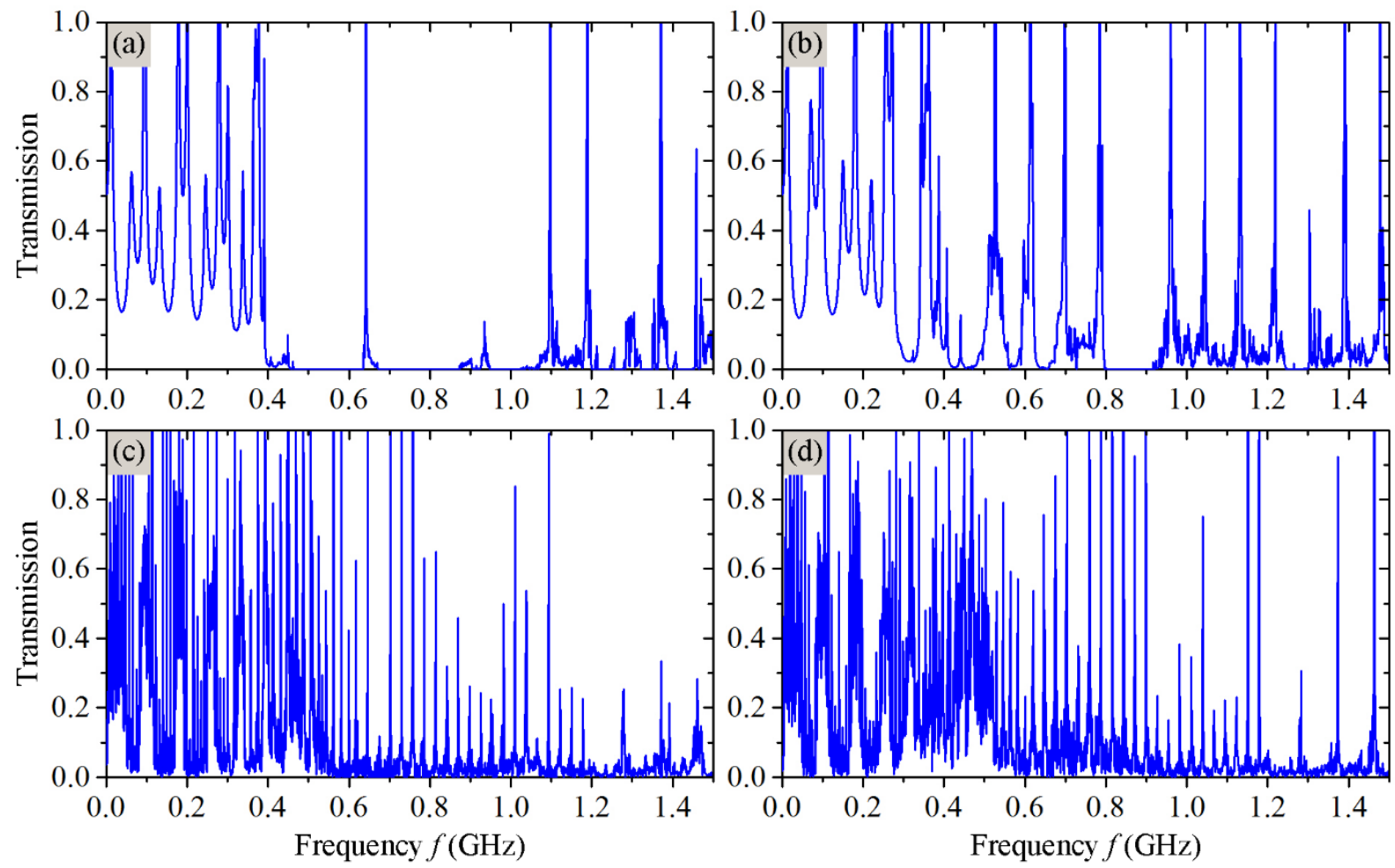
157 Fig.2. Transmission spectra of the proposed hierarchical composites with $N=1$ and $N=2$ level of 158 hierarchy. (a)-(b): Transmission spectra of nacre-like and biocalcite-like composites with $N=1$ level of 159 hierarchy; (c)-(d): Transmission spectra of nacre-like and biocalcite-like composites with $N=2$ levels of 160 hierarchy. The overall volume fractions of mineral phase in the composites with $N=1$ and $N=2$ levels of 161 hierarchy are equal to $V_{f n}=0.80$.

162

163 To validate our hypothesis of low frequency band gap formation, we calculate the phonon dispersion 164 relations of the two types of hierarchical composites with $N=2$ levels of hierarchy. To this end, a two165 dimensional supercell for each composite is considered to calculate the phonon dispersion relations 166 (Fig.1. (c) and (d)). Note that the normally incident elastic wave propagation in the hierarchical 167 composites corresponds to wave propagation along YГ direction of their first Brillouin zones [29]. 168 Figure 3(a) and (c) present the phonon dispersion relations of nacre-like and biocalcite-like composites 169 with $N=2$ levels of hierarchy, respectively. For the purpose of comparison, the transmission spectra 170 within $0 \sim 0.3 \mathrm{GHz}$ are also displayed (Fig.3. (b) and (d)). One notices that multiple partial band gaps 171 arise in the dispersion relations, which agree well with the attenuation zones in the corresponding 172 transmission spectra. This result supports that the low frequency band gaps stem from the structural 173 periodicity at level $n=2$ of the hierarchical composites. It is important to note that this low frequency 174 feature enables the design of compact and lightweight wave filters, thus providing more flexibility in 175 engineering practice.

To gain a better understanding of the low frequency band gap formation, we plot in Figure 4 the total displacement fields of the composites with $N=2$ levels of hierarchy under incident wave frequencies within the low frequency band gaps, as well as frequencies at passbands. At frequencies $f=0.079 \mathrm{GHz}$ and $f=0.071 \mathrm{GHz}$, within the band gaps of nacre-like and biocalcite-like composites, respectively, the incident wave is mostly reflected by the hierarchical composites, which is a strong evidence of Bragg scattering (Fig. 4. (a) and (c)). By contrast, at frequencies of passbands, the normally incident wave can pass through both of the hierarchical composites without decay. These phenomena suggest that the low 184 frequency band gaps are Bragg-type, where the wavelengths of elastic wave have the same order of 185 magnitude as the structural periodicities at level $n=2$ of the hierarchical composites. It is interesting to 186 note that the widths of these low frequency band gaps are much narrower than those of the continuous 187 band gaps in hierarchical composites with $N=1$ level of hierarchy. We believe that this is mainly because 
188 the contrast in elastic constants at hierarchical level $n=2$ is significantly reduced when the structural 189 hierarchy is increased from $N=1$ to $N=2$ [29]. To demonstrate this, we evaluate the stiffness contrasts of 190 the hierarchical composite with a nacre-like architecture, as an example. For $N=1$, the stiffness contrast 191 between mineral phase and organic phase is given by $\gamma_{N=1}=E_{m 1} / E_{o}=100$, whereas for $N=2$, this 192 contrast becomes $\gamma_{N=2}=E_{m 2} / E_{o}=30.8$, where $E_{o}=1 \mathrm{GPa}, E_{m 1}=E_{m}=100 \mathrm{GPa}$, and $E_{m 2}=30.8 \mathrm{GPa}$ is 193 estimated by the shear lag model [31]. Intrinsically, the stiffness contrast is solely dictated by the 194 geometric features of the nacre-like architecture including level of structural hierarchy, volume fraction 195 and lattice angle. This finding not only provides us a better understanding of the mechanisms accounting 196 for the low frequency band gaps, but also suggests possible avenues to design tunable phononic crystals 197 with optimal band gaps.

198
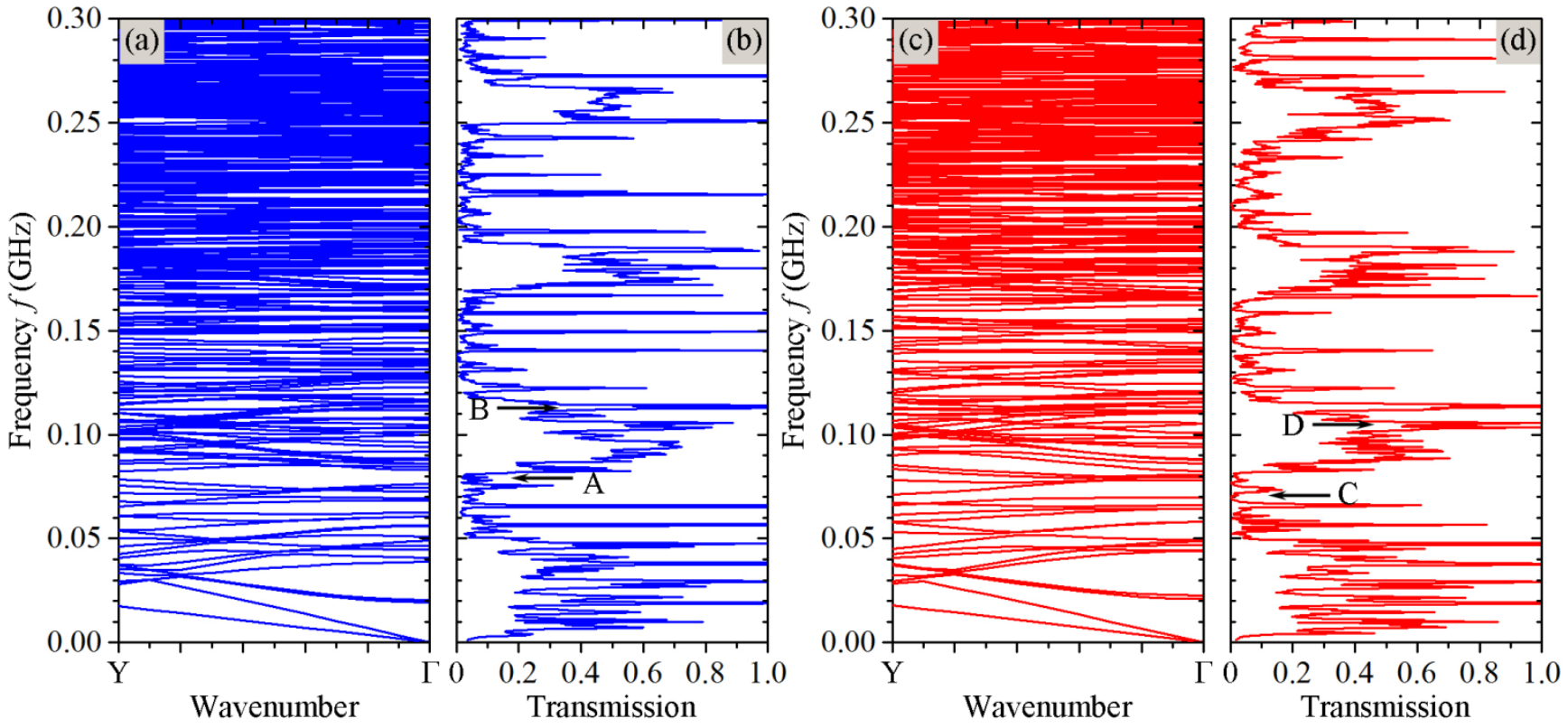

Fig.3. (a) and (c): phonon dispersion relations of nacre-like and biocalcite-like hierarchical composites at level $n=2$ along $\mathrm{Y} \Gamma$ direction, where $\mathrm{Y}=\left(0, \frac{\pi}{\left(l_{n}+t_{n}\right)} \cdot\left(\cot \frac{\alpha_{n}}{2}+\tan \frac{\alpha_{n}}{2}\right)\right)$, and $\Gamma=(0,0)[29]$; (b) and (d):

The corresponding transmission spectra of nacre-like and biocalcite-like composites with $N=2$ levels of hierarchy. 


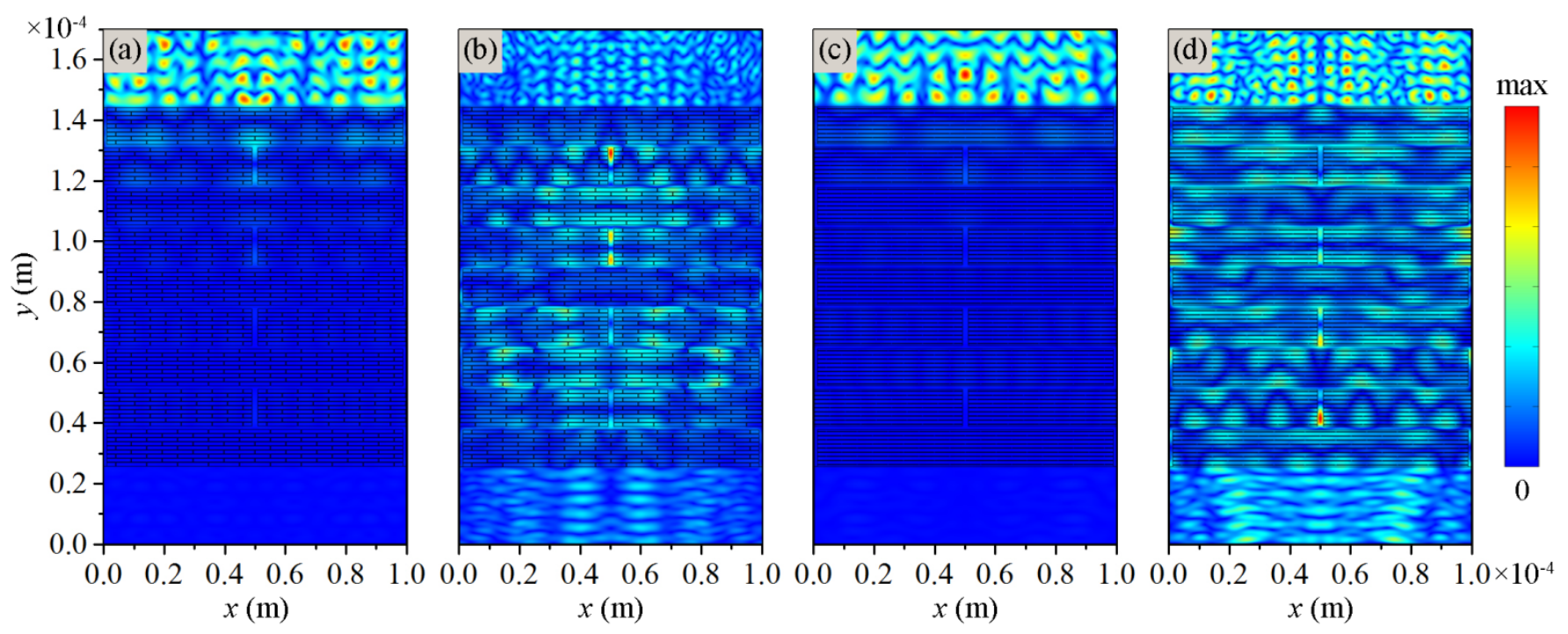

Fig. 4. Total displacement fields of hierarchical composites with $N=2$ levels of hierarchy at (a) $f=0.079$ $\mathrm{GHz}$, inside the band gap, and (b) $f=0.113 \mathrm{GHz}$, at passband for nacre-like composites; (c) $f=0.071 \mathrm{GHz}$, inside the band gap and (d) $f=0.105 \mathrm{GHz}$, at passband for biocalcite-like composite. The positions of the selected frequencies for (a)-(d) are labelled as A, B, C and D in Figure 3, respectively.

Having revealed the mechanisms responsible for the low frequency band gaps, we next explore the origin of multiband feature of the composites with $N=2$ levels of hierarchy. Here the band gaps and passbands within high frequency range $0.4 \sim 1.5 \mathrm{GHz}$ are particularly interested. A direct comparison of transmission spectra between composites with $N=1$ and $N=2$ levels of structural hierarchy inspire us to postulate that the interrupted periodicity at hierarchical level $n=1$ is responsible for the multiple sharp band gaps and passbands in the composites with $N=2$ levels of hierarchy. Indeed, the hierarchical composites with $N=2$ levels of hierarchy can be considered as two-dimensional periodic composites with defects. To validate this hypothesis, we calculate the transmission spectra of periodic composites without being interrupted, which is equivalent to the hierarchical composites with $N=1$ level of hierarchy, except that here the volume fraction of the hard mineral phase is $v_{f n}=0.894$ (Fig.5.(a) and (b)). As expected, the perfectly periodic composites exhibit wide continuous band gaps, as compared to the multiple sharp band gaps and passbands of composites with $N=2$ levels of hierarchy. This phenomenon further supports our claim that the structural hierarchy itself is responsible for the multiband feature. In other words, the defects, acting as waveguides in the composites with $N=2$ levels of hierarchy, give rise to multiple band gaps and passbands. 
227 To demonstrate that the waveguide modes are responsible for the multiple band gaps and passbands at 228 high frequency range, we present the total displacement fields of the composites with $N=2$ levels of 229 hierarchy at these high frequencies. Note that for the purpose of comparison, the frequencies of selected 230 band gaps and passbands also locate inside the band gaps of the perfectly periodic composites. At 231 frequencies $f=0.957 \mathrm{GHz}$ and $f=0.968 \mathrm{GHz}$, inside the band gaps, the elastic wave is well confined 232 within the waveguides. This is because the wave propagation is prohibited by the surrounding perfectly 233 periodic composites (Fig. 6. (a) and (c)). At passbands, as indicated in Fig. 6. (b) and (d), the incident 234 elastic wave is concentrated in the waveguides and transmits through the waveguides, and then radiates 235 at the exits of the waveguides. Although some of the wave energy penetrates into the surrounding 236 supercells, as indicated in Fig. $6\left(d_{1}\right)$, we believe this energy only takes a small proportion and most of 237 the concentrated wave energy transmits through the waveguide efficiently. This is supported by the high 238 transmission coefficient (0.75) of the elastic wave at $f=1.039 \mathrm{GHz}$, which corresponds to a waveguide 239 mode. Apparently, the physical mechanisms of multiple band gaps and passbands at high frequencies are 240 totally different from those of low frequency band gaps. We emphasize that the high frequency band 241 gaps and passbands of the hierarchical composites with $N=2$ levels of hierarchy correspond to 242 waveguide modes, which result from the introduction of defects; whereas the continuous band gaps in 243 the perfectly periodic composites and hierarchical composites with $N=1$ level of hierarchy are attributed 244 245 to Bragg scattering or local resonances [29]. It should be pointed out that the proposed hierarchical architectures with multilevel structural hierarchies also endow the composites with enhanced mechanical 246 properties including high strength and high fracture toughness [31-33]. This additionally exceptional 247 feature together with the wave filtering and waveguiding capabilities implies the possibility to design 248 phononic crystals suitable for mechanically challenging environmental conditions. 

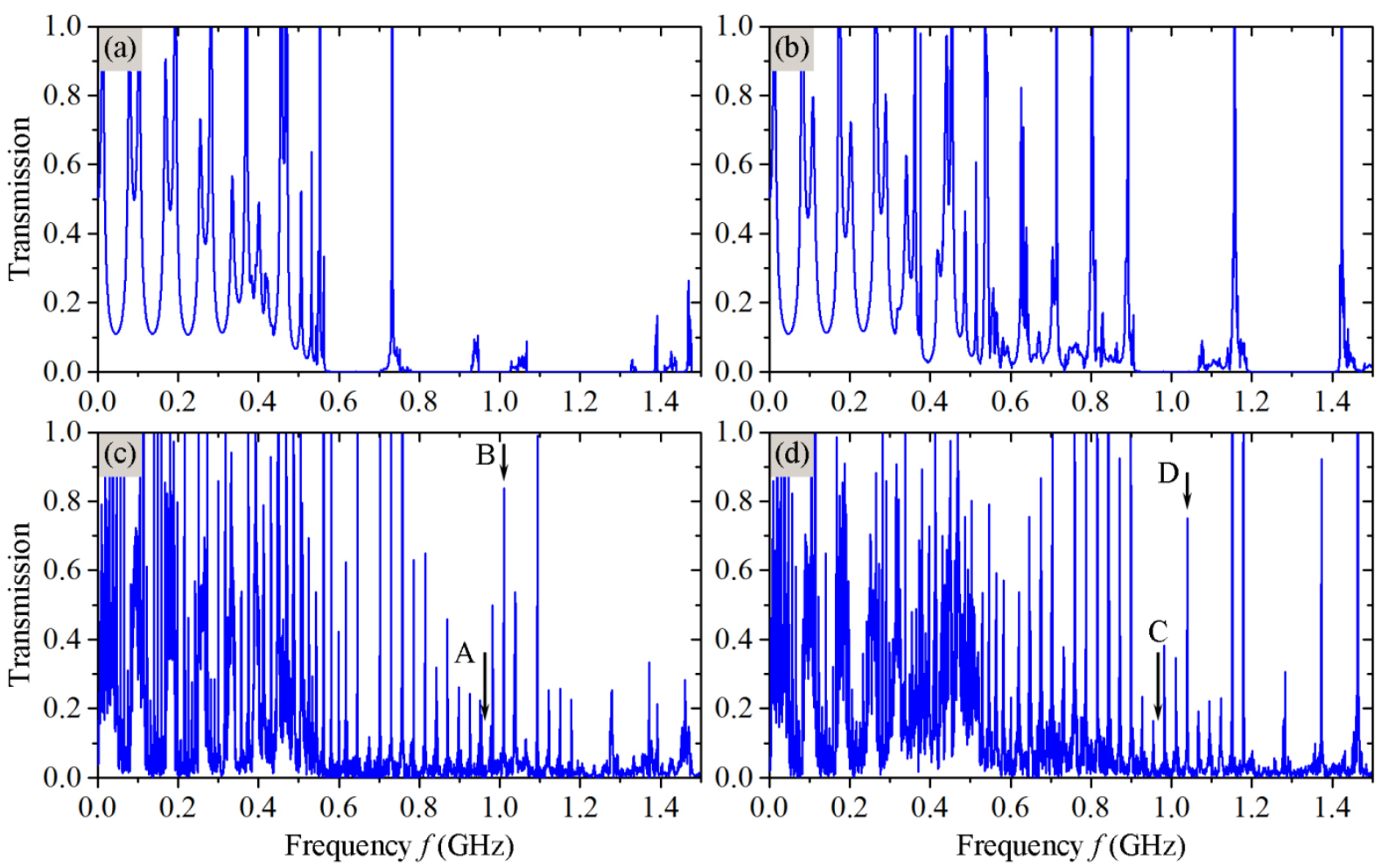

Fig. 5. (a)-(b): Transmission spectra of nacre-like and biocalcite-like perfectly periodic composites 252 without being interrupted, the unit cells of which are the same as those at level $n=1$ of composites with $253 N=2$ levels of hierarchy, except that here the volume fraction of the hard mineral phase is $v_{f n}=0.894$; (c)254 (d): Transmission spectra of nacre-like and biocalcite-like composites with $N=2$ levels of hierarchy. 

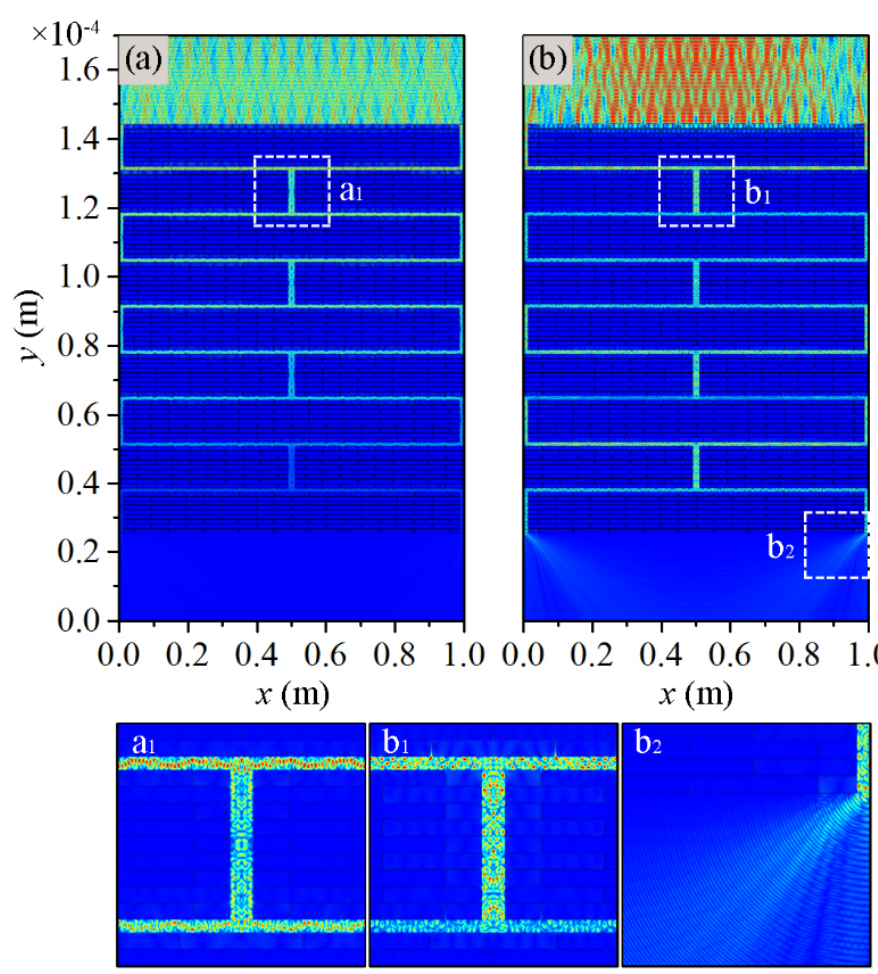
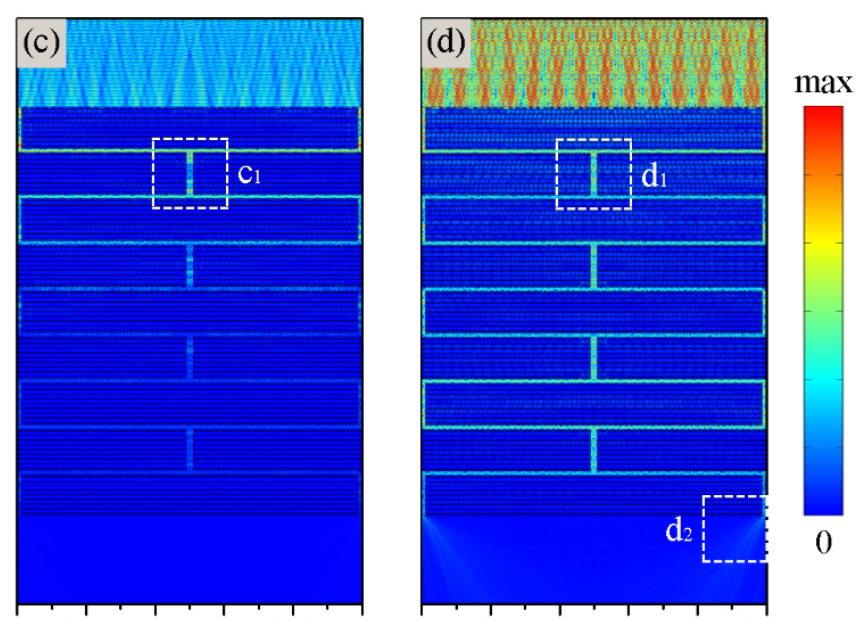

$\mathrm{b}_{2}$

Fig. 6. Total displacement fields of hierarchical composites with $N=2$ levels of hierarchy at (a) $f=0.957$ $\mathrm{GHz}$, inside the band gap, and (b) $f=1.010 \mathrm{GHz}$, at passband for nacre-like composites; (c) $f=0.968 \mathrm{GHz}$, inside the band gap and (d) $f=1.039 \mathrm{GHz}$, at passband for biocalcite-like composite. The positions of the selected frequencies for (a)-(d) are labelled as A, B, C and D in Figure 5, respectively.

\section{Conclusions}

In summary, we have investigated the elastic wave propagation in bio-inspired hierarchical composites with nacre-like and biocalcite-like architectures. Multiple band gaps and passbands arise in the proposed hierarchical composites with two levels of hierarchy. Remarkably, the low frequency band gaps, akin to the subwavelength characteristic of acoustic metamaterials, are attributed to the periodic arrangement of mineral and organic phases at the second hierarchical level. By contrast, the band gaps and passbands in high frequency ranges correspond to the waveguide modes, enabling the incident wave can be either well trapped inside the waveguides or efficiently transmit through the waveguides. These features enable the design of compact and lightweight wave filters and waveguides operating in an ultrawide frequency range. Notably, the hierarchical architectures with multilevel structural hierarchies also endow the proposed composites with enhanced strength and toughness, which are highly desirable for phononic crystals under mechanically challenging environmental conditions. The findings in this paper not only provide us a better understanding of the mechanisms accounting for the multiband features of bio- 
inspired hierarchical composites, but also offer new opportunities towards the design of compact and mechanically robust phononic crystals with capabilities to effectively manipulate wave propagation.

\section{Acknowledgements}

280 This work is partially supported by the National Science Foundation. L. Wang acknowledges start-up 281 funds from College of Engineering and Applied Sciences at Stony Brook University.

\section{References}

[1] Lee JH, Koh CY, Singer JP, Jeon SJ, Maldovan M, Stein O, et al. 25th Anniversary Article: Ordered polymer structures for the engineering of photons and phonons. Advanced Materials. 2014;26:532-69.

[2] Maldovan M. Sound and heat revolutions in phononics. Nature. 2013;503:209-17.

[3] Khelif A, Djafari-Rouhani B, Vasseur J, Deymier P. Transmission and dispersion relations of perfect and defect-containing waveguide structures in phononic band gap materials. Physical Review B. 2003;68:024302.

[4] Khelif A, Choujaa A, Djafari-Rouhani B, Wilm M, Ballandras S, Laude V. Trapping and guiding of acoustic waves by defect modes in a full-band-gap ultrasonic crystal. physical Review B. 2003;68:214301.

[5] Khelif A, Choujaa A, Benchabane S, Djafari-Rouhani B, Laude V. Guiding and bending of acoustic waves in highly confined phononic crystal waveguides. Applied Physics Letters. 2004;84:4400-2.

[6] Sun J-H, Wu T-T. Propagation of surface acoustic waves through sharply bent two-dimensional phononic crystal waveguides using a finite-difference time-domain method. Physical Review B. 2006;74:174305.

[7] Benchabane S, Gaiffe O, Salut R, Ulliac G, Laude V, Kokkonen K. Guidance of surface waves in a micron-scale phononic crystal line-defect waveguide. Applied Physics Letters. 2015;106:081903.

[8] Sun J-H, Wu T-T. Propagation of acoustic waves in phononic-crystal plates and waveguides using a finite-difference time-domain method. Physical Review B. 2007;76:104304.

[9] Pennec Y, Vasseur JO, Djafari-Rouhani B, Dobrzyński L, Deymier PA. Two-dimensional phononic crystals: Examples and applications. Surface Science Reports. 2010;65:229-91.

[10] Kushwaha MS. Stop-bands for periodic metallic rods: Sculptures that can filter the noise. Applied Physics Letters. 1997;70:3218-20.

[11] Vasseur J, Deymier P, Chenni B, Djafari-Rouhani B, Dobrzynski L, Prevost D. Experimental and theoretical evidence for the existence of absolute acoustic band gaps in two-dimensional solid phononic crystals. Physical Review Letters. 2001;86:3012.

[12] Pennec Y, Djafari-Rouhani B, Vasseur J, Khelif A, Deymier P. Tunable filtering and demultiplexing in phononic crystals with hollow cylinders. Physical Review E. 2004;69:046608.

[13] Hou B, Xie H, Wen W, Sheng P. Three-dimensional metallic fractals and their photonic crystal characteristics. Physical Review B. 2008;77:125113.

[14] Wen W, Zhou L, Li J, Ge W, Chan C, Sheng P. Subwavelength photonic band gaps from planar fractals. Physical review letters. 2002;89:223901.

[15] Zhou L, Chan C, Sheng P. Theoretical studies on the transmission and reflection properties of metallic planar fractals. Journal of Physics D: Applied Physics. 2004;37:368. 
[16] Chen D, Wang S, Li L, Liu Z, Zhao X-Z, Zhang M, et al. Microstrip filter with H-shaped fractal. Applied physics letters. 2006;88:253507. Theoretical study using numerical analysis. Journal of Applied Physics. 2008;103:104908.

[18] Kuo N-K, Piazza G. Fractal phononic crystals in aluminum nitride: An approach to ultra high frequency bandgaps. Applied Physics Letters. 2011;99:163501.

[19] Xiao-Jian L, You-Hua F. Band structure characteristics of T-square fractal phononic crystals. Chinese Physics B. 2013;22:036101.

[20] Zhang P, To AC. Broadband wave filtering of bioinspired hierarchical phononic crystal. Applied physics letters. 2013;102:121910.

[21] Hussein MI, Hulbert GM, Scott RA. Dispersive elastodynamics of 1D banded materials and structures: design. Journal of Sound and Vibration. 2007;307:865-93.

[22] Hussein MI, Hamza K, Hulbert GM, Scott RA, Saitou K. Multiobjective evolutionary optimization of periodic layered materials for desired wave dispersion characteristics. Structural and Multidisciplinary Optimization. 2006;31:60-75.

[23] Ji B, Gao H. Mechanical properties of nanostructure of biological materials. Journal of the Mechanics and Physics of Solids. 2004;52:1963-90.

[24] Bertoldi K, Bigoni D, Drugan W. Nacre: an orthotropic and bimodular elastic material. Composites Science and Technology. 2008;68:1363-75.

[25] Dashkovskiy S, Suhr B, Tushtev K, Grathwohl G. Nacre properties in the elastic range: Influence of matrix incompressibility. Computational Materials Science. 2007;41:96-106.

[26] Wang R, Suo Z, Evans A, Yao N, Aksay I. Deformation mechanisms in nacre. Journal of Materials Research. 2001;16:2485-93.

[27] Barthelat F, Li C-M, Comi C, Espinosa HD. Mechanical properties of nacre constituents and their impact on mechanical performance. Journal of Materials Research. 2006;21:1977-86.

[28] Khelif A, Achaoui Y, Benchabane S, Laude V, Aoubiza B. Locally resonant surface acoustic wave band gaps in a two-dimensional phononic crystal of pillars on a surface. Physical Review B. 2010;81:214303.

[29] Chen Y, Wang L. Tunable band gaps in bio-inspired periodic composites with nacre-like microstructure. Journal of Applied Physics. 2014;116:063506.

[30] Liu Z, Zhang X, Mao Y, Zhu Y, Yang Z, Chan C, et al. Locally resonant sonic materials. Science. 2000;289:1734-6.

[31] Yao H, Gao H. Reprint of "Multi-scale cohesive laws in hierarchical materials"[In. J. Solids Struct. 44 (2007) 8177-8193]. International Journal of Solids and Structures. 2008;45:3627-43.

[32] Bechtle S, Ang SF, Schneider GA. On the mechanical properties of hierarchically structured biological materials. Biomaterials. 2010;31:6378-85.

[33] Sen D, Buehler MJ. Structural hierarchies define toughness and defect-tolerance despite simple and mechanically inferior brittle building blocks. Scientific reports. 2011;1. 